Internat. J. Math. \& Math. Sci.

Vol. 23, No. 4 (2000) 271-277

S0161171200000764

(C) Hindawi Publishing Corp.

\title{
INFINITE PRODUCTS OVER HYPERPYRAMID LATTICES
}

\author{
GEOFFREY B. CAMPBELL
}

(Received 2 July 1996)

\begin{abstract}
New infinite product identities are given, based on summed visible (from the origin) point vectors. Each result is found from summing on vpv lattices dividing space into radial regions from the origin. Recently, Baake et al. and Mosseri considered the 2-D visible lattice points as part of an optical experiment in which so-called Optical Fourier Transform was applied. Many of the techniques exposed by Glasser and Zucker, and Ninham et al. involving Mellin and Möbius inversions are also applicable to the current paper.
\end{abstract}

Keywords and phrases. Combinatorial identities, combinatorial number theory, lattice points in specified regions, partitions.

2000 Mathematics Subject Classification. Primary 05A19, 11B75, 11P21, 11P81.

1. A hyperpyramid theorem. In five recent papers by the author $[8,6,7,9,5]$, some new infinite product identities were given. These were called visible (from the origin) point vector identities, or simply $v p v$ identities. Each result was found from summing on vpv lattices dividing space into radial regions from the origin. Recently also (see Baake et al. [3] and Mosseri [12]) the visible lattice points were considered as part of an optical experiment in which the so-called Optical Fourier Transform was applied to the 2-D vpv's. Apostol [2] gave an excellent introduction to the ideas behind vpv's and calculated their density in space. Many of the techniques exposed in Glasser and Zucker [10], and in Ninham et al. [13] involving Mellin and Möbius inversions are applicable to vpv identities from the author's papers $[8,6,7,9,5]$ and to the current paper. In Andrew's book on partitions [1], a historical perspective of generating functions for partitions, and an introduction to the works on plane partitions and vector partitions were given. The identities in the papers $[8,6,7,9,5]$ and in the current paper are related to ideas from this book. Each vpv identity turns out to be combinatorially equivalent to a theorem on weighted vector partitions (see [6]). Here, we give two analogous theorems to the general one appearing in [7].

THEOREM 1.1. If $i=1,2, \ldots, n$; then, for each $\left|x_{n}\right|,\left|x_{n} x_{n-1}\right|, \ldots,\left|x_{n} x_{n-1} \cdots x_{1}\right|<1$, and $b_{i} \in C$,

$$
\begin{aligned}
\exp \left\{\sum_{k \geq 1} \frac{x_{n}^{k}}{k^{b_{n}}}\right\} \prod_{\substack{\left(a_{1}, \ldots, a_{n}\right)=1 \\
a_{1}, \ldots, a_{n-1}<a_{n} \\
a_{1}, \ldots, a_{n-1}>0 \\
a_{n}>1}}\left(1-x_{1}^{a_{1}} \cdots x_{n}^{a_{n}}\right)^{-a_{1}^{-b_{1}} \cdots a_{n}^{-b_{n}}} \\
\quad=\exp \left\{\sum_{k=1}^{\infty} \prod_{i=1}^{n-1}\left(\sum_{j=1}^{k-1} \frac{x_{i}^{j}}{j^{b_{i}}}\right) \frac{x_{n}^{k}}{k^{b_{n}}}\right\}, \quad \text { provided } \sum_{i=1}^{n} b_{i}=1 .
\end{aligned}
$$


This result is quite easy to prove using a technique in Campbell $[8,6,7,9,5]$ by summing on the vpv's in the $n$-space hyperpyramid, defined by the inequalities

$$
x_{1}<x_{n}, x_{2}<x_{n}, \ldots, x_{n-1}<x_{n}
$$

in the first $n$-space hyperquadrant, and applying Lemma 1.6 below.

The corresponding theorem from Campbell [7] was summed very simply over all lattice point vectors in the first hyperquadrant. The approach we adopt to give the reader a feel for these identities is to take corollaries and then examples from them. The 2-D case through to the 5-D case of (1.1) are given in the following corollaries.

COROLLARY 1.2. If $|y z|$ and $|z|<1$, and $S+t=1$, then

$$
\begin{aligned}
\exp \left\{\sum_{k \geq 1} \frac{z^{k}}{k^{t}}\right\} & \prod_{\substack{(a, b)=1 \\
a<b \\
a>0 ; b<1}}\left(1-y^{a} z^{b}\right)^{-a^{-s} b^{-t}} \\
& =\exp \left\{\frac{z^{1}}{1^{t}}+\left(1+\frac{y^{1}}{1^{s}}\right) \frac{z^{2}}{2^{t}}+\left(1+\frac{y^{1}}{1^{s}}+\frac{y^{2}}{2^{s}}\right) \frac{z^{3}}{3^{t}}+\cdots\right\} .
\end{aligned}
$$

COROLLARY 1.3. If $|x y z|,|y z|$, and $|z|<1$, and $r+s+t=1$, then

$$
\begin{aligned}
& \exp \left\{\sum_{k \geq 1} \frac{z^{k}}{k^{t}}\right\} \prod_{\substack{(a, b, c)=1 \\
a, b<c \\
a, b>0 ; c<1}}\left(1-x^{a} y^{b} z^{c}\right)^{-a^{-r} b^{-s} c^{-t}} \\
& =\exp \left\{\frac{z^{1}}{1^{t}}+\left(1+\frac{x^{1}}{1^{r}}\right)\left(1+\frac{y^{1}}{1^{s}}\right) \frac{z^{2}}{2^{t}}+\left(1+\frac{x^{1}}{1^{r}}+\frac{x^{2}}{2^{r}}\right)\left(1+\frac{y^{1}}{1^{s}}+\frac{y^{2}}{2^{s}}\right) \frac{z^{3}}{3^{t}}+\cdots\right\} .
\end{aligned}
$$

COROLLARY 1.4. If $|w x y z|,|x y z|,|y z|$, and $|z|<1$, and $q+r+s+t=1$, then

$$
\begin{aligned}
\exp \left\{\sum_{k \geq 1} \frac{z^{k}}{k^{t}}\right\} \prod_{\substack{(a, b, c, d)=1 \\
a, b, c<d \\
a, b, c>0 ; d>1}}\left(1-w^{a} x^{b} y^{c} z^{d}\right)^{-a^{-q} b^{-r} c^{-s} d^{-t}} \\
=\exp \left\{\frac{z^{1}}{1^{t}}+\left(1+\frac{w^{1}}{1^{q}}\right)\left(1+\frac{x^{1}}{1^{r}}\right)\left(1+\frac{y^{1}}{1^{s}}\right) \frac{z^{2}}{2^{t}}\right. \\
\left.\quad+\left(1+\frac{w^{1}}{1^{q}}+\frac{w^{2}}{2^{q}}\right)\left(1+\frac{x^{1}}{1^{r}}+\frac{x^{2}}{2^{r}}\right)\left(1+\frac{y^{1}}{1^{s}}+\frac{y^{2}}{2^{s}}\right) \frac{z^{3}}{3^{t}}+\cdots\right\} .
\end{aligned}
$$

COROLLARY 1.5. If $|v w x y z|,|w x y z|,|x y z|,|y z|$, and $|z|<1$, and $p+q+r+s+$ $t=1$, then 


$$
\begin{aligned}
\exp \left\{\sum_{k \geq 1} \frac{z^{k}}{k^{t}}\right\} \prod_{\substack{a, b, c, d, e)=1 \\
a, b, c, d<e \\
a, b, c, d>0 ; e>1}}\left(1-v^{a} w^{b} x^{c} y^{d} z^{e}\right)^{-a^{-p} b^{-q} c^{-r} d^{-s} e^{-t}} \\
=\exp \left\{\frac{z^{1}}{1^{t}}+\left(1+\frac{v^{1}}{1^{p}}\right)\left(1+\frac{w^{1}}{1^{q}}\right)\left(1+\frac{x^{1}}{1^{r}}\right)\left(1+\frac{y^{1}}{1^{s}}\right) \frac{z^{2}}{2^{t}}\right. \\
\left.+\left(1+\frac{v^{1}}{1^{p}}+\frac{v^{2}}{2^{p}}\right)\left(1+\frac{w^{1}}{1^{q}}+\frac{w^{2}}{2^{q}}\right)\left(1+\frac{x^{1}}{1^{r}}+\frac{x^{2}}{2^{r}}\right)\left(1+\frac{y^{1}}{1^{s}}+\frac{y^{2}}{2^{s}}\right) \frac{z^{3}}{3^{t}}+\cdots\right\} .
\end{aligned}
$$

The above results can all be proved easily when forming multiple sums derived from the logarithm of both sides, and applying the following lemma.

LEMMA 1.6. Consider an infinite region raying out of the origin in any Euclidean vector space. The set of all lattices point vectors from the origin in that region is precisely the set of positive integer multiples of the visible point vectors ( $v p v$ 's) in that region.

2. Examples of the corollaries. The simplest examples are obtained by setting $t=1$ (hence, $p, q, r$, and $s=0$ ) in the above, yielding

$$
\begin{gathered}
\prod_{\substack{a, b)=1 \\
a<b \\
a \geq 0 ; b \geq 1}}\left(1-y^{a} z^{b}\right)^{-b^{-1}}=\left(\frac{1-y z}{1-z}\right)^{1 /(1-y)} \\
\prod_{\substack{a, b, c)=1 \\
a, b<c \\
a, b \geq 0 ; c \geq 1}}\left(1-x^{a} y^{b} z^{c}\right)^{-c^{-1}}=\left(\frac{(1-x z)(1-y z)}{(1-z)(1-x y z)}\right)^{1 /((1-x)(1-y))} \\
\prod_{\substack{a, b, c, d)=1 \\
a, b, c<d \\
a, b, c \geq 0 ; d \geq 1}}\left(1-w^{a} x^{b} y^{c} z^{d}\right)^{-d^{-1}} \\
=\left(\frac{(1-w z)(1-x z)(1-y z)(1-w x y z)}{(1-z)(1-w x z)(1-w y z)(1-x y z)}\right)^{1 /((1-w)(1-x)(1-y))} \\
\prod_{\substack{(a, b, c, d, e)=1 \\
a, b, c, d<e \\
a, b, c, d \geq 0 ; e \geq 1}}^{\left(1-v^{a} w^{b} x^{c} y^{d} z^{e}\right)^{-e^{-1}}} \\
=\left(\begin{array}{l}
(1-v z)(1-w z)(1-x z)(1-y z) \\
(1-z)(1-v w z)(1-v x z)(1-v y z)
\end{array}\right. \\
\left.\times \frac{(1-v w y z)(1-v x y z)(1-w x y z)}{(1-w x z)(1-w y z)(1-x y z)(1-v w x y z)}\right)^{1 /((1-v)(1-w)(1-x)(1-y))}
\end{gathered}
$$

These results were given in Campbell $[6,7]$ without the full generality of Theorem 1.1 nor of the corollaries. Particular cases of (2.1), (2.2), (2.3), and (2.4) were examined in 
Campbell [9], where they were shown to have nontrivial simple cases. For example, if each of $v, w, x$, and $y$ are set equal to $z$, then the binomial coefficients appear as exponents in the right-hand sides of (2.1), (2.2), (2.3), and (2.4), and (2.4) say, becomes

$$
\prod_{\substack{a, b, c, d, e)=1 \\ a, b, c, d<e \\ a, b, c, d \geq 0 ; e \geq 1}}\left(1-z^{(a+b+c+d+e)}\right)^{-e^{-1}}=\left(\frac{\left(1-z^{2}\right)^{4}\left(1-z^{4}\right)^{4}}{(1-z)\left(1-z^{3}\right)^{6}\left(1-z^{5}\right)}\right)^{1 /(1-z)^{4}} .
$$

This particular case was not given previously. The right-hand side can be easily expanded into a power series in $z$ and the left-hand side, when expanded, enumerates weighted vpv vector partitions.

3. A general hyperpyramid theorem. In Campbell [7], the main theorem of the paper came from summing on all lattice points in the first hyperquadrant. In Section 1 , this restricted to hyperpyramid lattices with symmetry in $n-1$ out of the $n$ variables. This is evident by viewing Corollaries $1.2,1.3,1.4$, and 1.5 . If we choose to vary the shape of our hyperpyramid, the process is most easily illustrated as follows. Consider the variant of identity (1.5), where the $x$ variable in the lattice is confined as follows:

$$
\begin{aligned}
& \exp \left\{\sum_{k \geq 1} \frac{z^{k}}{k^{t}}\right\} \prod_{\substack{(a, b, c, d)=1 \\
a, b, c, c<d \\
a, b, c>0 ; d>1}}\left(1-w^{a} x^{b} y^{c} z^{d}\right)^{-a^{-a} b^{-r} c^{-s} d^{-t}} \\
& =\exp \left\{\left(1+\frac{x^{1}}{1^{r}}\right) \frac{z^{1}}{1^{t}}+\left(1+\frac{w^{1}}{1^{q}}\right)\left(1+\frac{x^{1}}{1^{r}}+\frac{x^{2}}{2^{r}}+\frac{x^{3}}{3^{r}}\right)\left(1+\frac{y^{1}}{1^{s}}\right) \frac{z^{2}}{2^{t}}\right. \\
& \left.+\left(1+\frac{w^{1}}{1^{q}}+\frac{w^{2}}{2^{q}}\right)\left(1+\frac{x^{1}}{1^{r}}+\frac{x^{2}}{2^{r}}+\frac{x^{3}}{3^{r}}+\frac{x^{4}}{4^{r}}+\frac{x^{5}}{5^{r}}\right)\left(1+\frac{y^{1}}{1^{s}}+\frac{y^{2}}{2^{s}}\right) \frac{z^{3}}{3^{t}}+\cdots\right\} .
\end{aligned}
$$

It becomes clear from this that the general hyperpyramid sums can be all included in the following theorem.

THEOREM 3.1. For conditions of Theorem 1.1, and in addition for $\alpha_{i} \in \mathfrak{R}^{+}$, we have

$$
\begin{aligned}
\exp \left\{\sum_{k \geq 1} \frac{z^{k}}{k^{t}}\right\} \prod_{\substack{\left(a_{1}, \ldots, a_{n}\right)=1 \\
\alpha_{1} a_{1}, \ldots, \alpha_{n-1} a_{n-1}<a_{n} \\
a_{1}, \ldots, a_{n-1}>0 \\
a_{n}>1}}\left(1-x_{1}^{a_{1}} \cdots x_{n}^{a_{n}}\right)^{-a_{1}^{-b_{1}} \cdots a_{n}^{-b_{n}}} \\
\quad=\exp \left\{\sum_{k=1}^{\infty} \prod_{i=1}^{n-1}\left(\sum_{j=1}^{F(k)} \frac{x_{i}^{j}}{j^{b_{i}}}\right) \frac{x_{n}^{k}}{k^{b_{n}}}\right\}, \quad \text { provided } \sum_{i=1}^{n} b_{i}=1,
\end{aligned}
$$

where

$$
F(k)= \begin{cases}{\left[\frac{k-1}{\alpha_{i}}\right]} & \text { if } \alpha_{i}>1 \\ k-1 & \text { if } \alpha_{i}=1 \\ {\left[\frac{k}{\alpha_{i}}-1\right]} & \text { if } \alpha_{i}<1\end{cases}
$$


We see that this theorem counts terms on any possible hyperpyramid lattice in the first hyperquadrant, where the hyperpyramid apex is at the origin. We may allow interesting limits to apply such as $\alpha_{i} \rightarrow 0$ for a particular $\alpha$. This contributes a factor to the infinite series on the right side exponentiated sum (3.2) since $F(k)$ is arbitrarily large. If each of the $\alpha$ 's in the theorem are less than 1, the resulting identity is simpler than the case when some or all $\alpha$ 's are greater than 1 . Indeed, applying the above theorem in a similar fashion to that of Section 2, we easily arrive at examples such as:

$$
\begin{gathered}
\prod_{\substack{(a, b)=1 \\
a / 11<b \\
a \geq 0 ; b \geq 1}}\left(1-y^{a} z^{b}\right)^{-b^{-1}}=\left(\frac{1-y^{11} z}{1-z}\right)^{1 /(1-y)} \\
\prod_{\substack{(a, b, c)=1 \\
a / 5, b / 7<c \\
a, b \geq 0 ; c \geq 1}}\left(1-x^{a} y^{b} z^{c}\right)^{-c^{-1}}=\left(\frac{\left(1-x^{5} z\right)\left(1-y^{7} z\right)}{(1-z)\left(1-x^{5} y^{7} z\right)}\right)^{1 /((1-x)(1-y))} \\
\prod_{\substack{(a, b, c, d)=1 \\
a / 13, b, 6, c ; 17<d \\
a, b, c \geq 0 ; d \geq 1}}\left(1-w^{a} x^{b} y^{c} z^{d}\right)^{-d^{-1}} \\
=\left(\frac{\left(1-w^{13} z\right)\left(1-x^{6} z\right)\left(1-y^{17} z\right)\left(1-w^{13} x^{6} y^{17} z\right)}{(1-z)\left(1-w^{13} x^{6} z\right)\left(1-w^{13} y^{17} z\right)\left(1-x^{6} y^{17} z\right)}\right)^{1 /((1-w)(1-x)(1-y))} \\
\prod_{\substack{(a, b, c, d, e)=1 \\
a, b, 2, c, c, d / 4<e \\
a, b, c, d \geq 0 ; e \geq 1}}\left(1-v^{a} w^{b} x^{c} y^{d} z^{e}\right)^{-e^{-1}} \\
=\left(\frac{(1-v z)\left(1-w^{2} z\right)\left(1-x^{3} z\right)\left(1-y^{4} z\right)\left(1-v w^{2} x^{3} z\right)}{(1-z)\left(1-v w^{2} z\right)\left(1-v x^{3} z\right)\left(1-v y^{4} z\right)\left(1-w^{2} x^{3} z\right)}\right. \\
\left.\times \frac{\left(1-v w^{2} y^{4} z\right)\left(1-v x^{3} y^{4} z\right)\left(1-w^{2} x^{3} y^{4} z\right)}{\left(1-w^{2} y^{4} z\right)\left(1-x^{3} y^{4} z\right)\left(1-v w^{2} x^{3} y^{4} z\right)}\right)^{1 /((1-v)(1-w)(1-x)(1-y))}
\end{gathered}
$$

The analogy of (2.5) obtained from (3.7) is easily written as

$$
\prod_{\substack{(a, b, c, d, e)=1 \\ a, b / 2, c / 3, d / 4<e \\ a, b, c, d \geq 0 ; e \geq 1}}\left(1-z^{(a+b+c+d+e)}\right)^{-e^{-1}}=\left(\frac{\left(1-z^{2}\right)\left(1-z^{3}\right)\left(1-z^{9}\right)\left(1-z^{10}\right)}{(1-z)\left(1-z^{6}\right)^{2}\left(1-z^{11}\right)}\right)^{1 /(1-z)^{4}} .
$$

4. Outline of the proof of Theorem 3.1. This is substantially the same method given in Campbell [7]. We consider only the case of $n=2$ as it shows the method for the general case, and there is no problem in generalizing the process. We start with the sum valid for the criteria of the theorem

$$
\sum_{b \geq 1} \frac{z^{b}}{b^{t}}+\sum_{\substack{\alpha a<b \\ a>0 ; b \geq 2}} \frac{y^{a} z^{b}}{a^{s} b^{t}}=\sum_{k=1}^{\infty}\left(\sum_{j=1}^{F(k)} \frac{y^{j}}{j^{s}}\right) \frac{z^{j}}{k^{t}} .
$$


We note by illustration that this is easily seen, for $\alpha=1 / 2$, to be

$$
\frac{z^{1}}{1^{t}}+\frac{z^{2}}{2^{t}}\left(1+\frac{y^{1}}{1^{s}}\right)+\frac{z^{3}}{3^{t}}\left(1+\frac{y^{1}}{1^{s}}\right)+\frac{z^{4}}{4^{t}}\left(1+\frac{y^{1}}{1^{s}}\right)+\frac{z^{5}}{5^{t}}\left(1+\frac{y^{1}}{1^{s}}+\frac{y^{2}}{2^{S}}\right)+\cdots,
$$

or, for $\alpha=1 / 3$, we have (4.1) as

$$
\frac{z^{1}}{1^{t}}+\frac{z^{2}}{2^{t}}\left(1+\frac{y^{1}}{1^{s}}\right)+\frac{z^{3}}{3^{t}}\left(1+\frac{y^{1}}{1^{s}}\right)+\frac{z^{4}}{4^{t}}\left(1+\frac{y^{1}}{1^{s}}\right)+\frac{z^{5}}{5^{t}}\left(1+\frac{y^{1}}{1^{s}}\right)+\frac{z^{6}}{6^{t}}\left(1+\frac{y^{1}}{1^{s}}+\frac{y^{2}}{2^{s}}\right)+\cdots .
$$

Let $\lambda=s+t$. By Lemma 1.6, equation (4.1) can be written as

$$
\begin{aligned}
\left(\sum_{b \geq 1} \frac{z^{b}}{b}\right) & +\sum_{\substack{\alpha a<b \\
(a, b)=1 \\
a \geq 1 ; b \geq 2}}\left(\frac{\left(y^{a} z^{b}\right)^{1}}{1^{\lambda}}+\frac{\left(y^{a} z^{b}\right)^{2}}{2^{\lambda}}+\frac{\left(y^{a} z^{b}\right)^{3}}{3^{\lambda}}+\cdots\right) \frac{1}{a^{s} b^{t}} \\
& =-\log (1-z)+\sum_{\substack{\alpha a<b \\
a, b=1 \\
a \geq 1 ; b \geq 2}} \frac{-1}{a^{s} b^{t}} \log \left(1-y^{a} z^{b}\right)
\end{aligned}
$$

if and only if $\lambda=1$. Exponentiating both sides of each of (4.1) and (4.2) and then equating the right-hand sides of these yield the $n=2$ equivalent of Theorem 3.1. Cases of this with $\alpha=1$ and $\alpha=1 / 11$ yield (2.1) and (3.4), respectively.

ACKNOWLEDGEMENT. The author would like to thank the referees for their important comments.

\section{REFERENCES}

[1] G. E. Andrews, The Theory of Partitions, Addison-Wesley Publishing Co., Reading, Mass.London-Amsterdam, 1976. MR 58\#27738. Zbl 371.10001.

[2] T. M. Apostol, Introduction to Analytic Number Theory, Springer-Verlag, New York, 1976. MR 55\#7892. Zbl 335.10001.

[3] M. Baake, U. Grimm, and D. H. Warrington, Some remarks on the visible points of a lattice, J. Phys. A 27 (1994), no. 8, 2669-2674. MR 95e:11071a. Zbl 960.09433.

[4] J. M. Borwein and P. B. Borwein, Pi and the AGM, John Wiley \& Sons Inc., New York, 1987. MR 89a:11134. Zbl 611.10001.

[5] G. B. Campbell, Multiplicative functions from Riemann zeta function products, J. Ramanujan Math. Soc. 7 (1992), no. 1, 53-63. MR 93m:11083. Zbl 753.11001.

[6]_ Dirichlet summations and products over primes, Internat. J. Math. Math. Sci. 16 (1993), no. 2, 359-372. MR 94c:11082. Zbl 776.11046.

[7] _ Infinite products over visible lattice points, Internat. J. Math. Math. Sci. 17 (1994), no. 4, 637-654. MR 95h:11105. Zbl 811.05006.

[8] _ A new class of infinite products, and Euler's totient, Internat. J. Math. Math. Sci. 17 (1994), no. 3, 417-422. MR 95d:11141. Zbl 809.05010.

[9] _ A closer look at some new identities, Internat. J. Math. Math. Sci. 21 (1998), no. 3, 581-586. MR 99d:11110. Zbl 908.05009.

[10] M. L. Glasser and I. J. Zucker, Lattice sums, Theoretical chemistry, Adv. and perspect 5 (1980), 67-139. Zbl 638.33007.

[11] G. H. Hardy and E. M. Wright, An Introduction to the Theory of Numbers, The Clarendon Press Oxford University Press, New York, 1979. MR 81i:10002. Zbl 423.10001.

[12] R. Mosseri, Visible points in a lattice, J. Phys. A 25 (1992), no. 1, L25-L29. MR 93b:52018. Zbl 771.11026. 
[13] B. W. Ninham, B. D. Hughes, N. E. Frankel, and M. L. Glasser, Möbius, Mellin, and mathematical physics, Phys. A 186 (1992), no. 3-4, 441-481. MR 93h:11143.

CAmpbell: Centre for Mathematics and its Applications, School of Mathematical SCienCes, Australian National University, CANBERRA, ACT 0200, Australia 


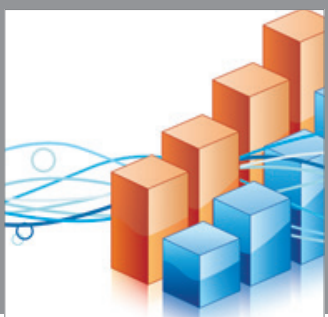

Advances in

Operations Research

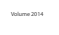

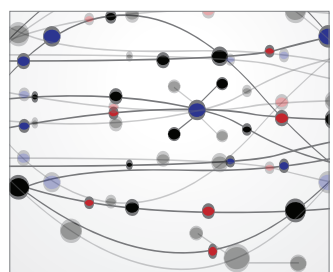

\section{The Scientific} World Journal
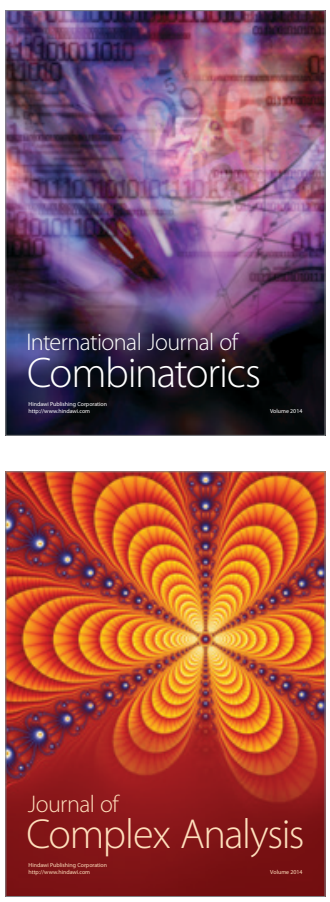

International Journal of

Mathematics and

Mathematical

Sciences
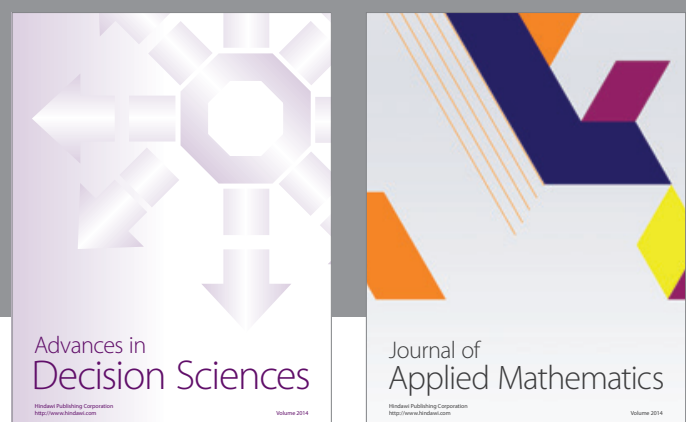

Journal of

Applied Mathematics
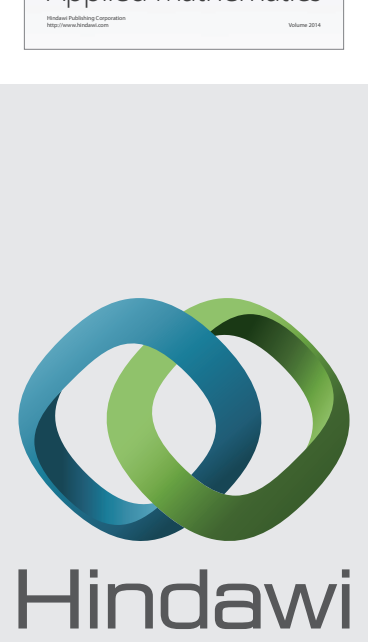

Submit your manuscripts at http://www.hindawi.com
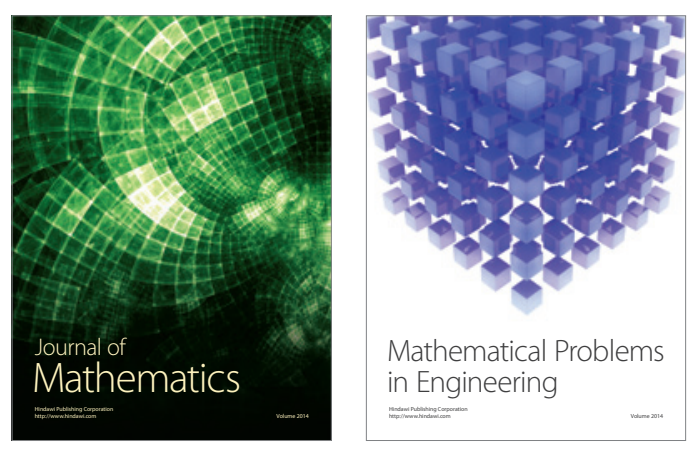

Mathematical Problems in Engineering
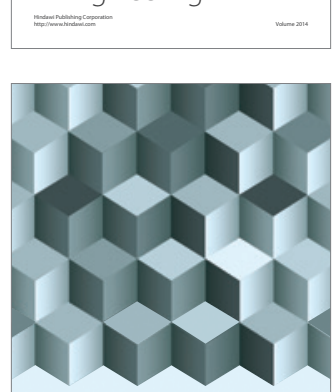

Journal of

Function Spaces
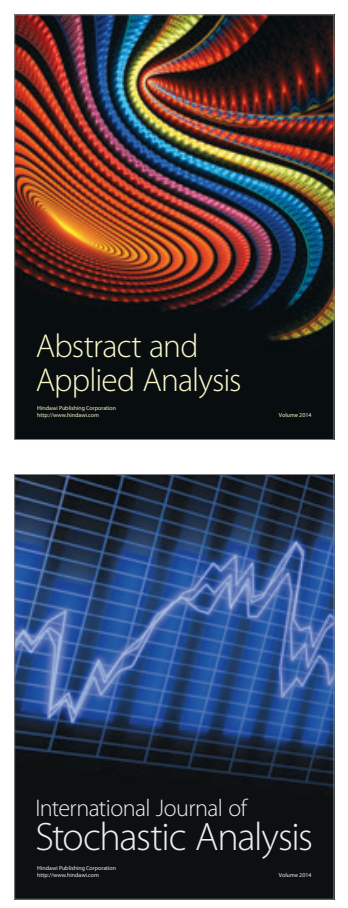

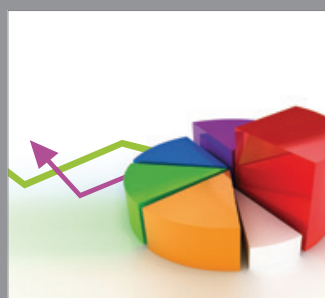

ournal of

Probability and Statistics

Promensencen
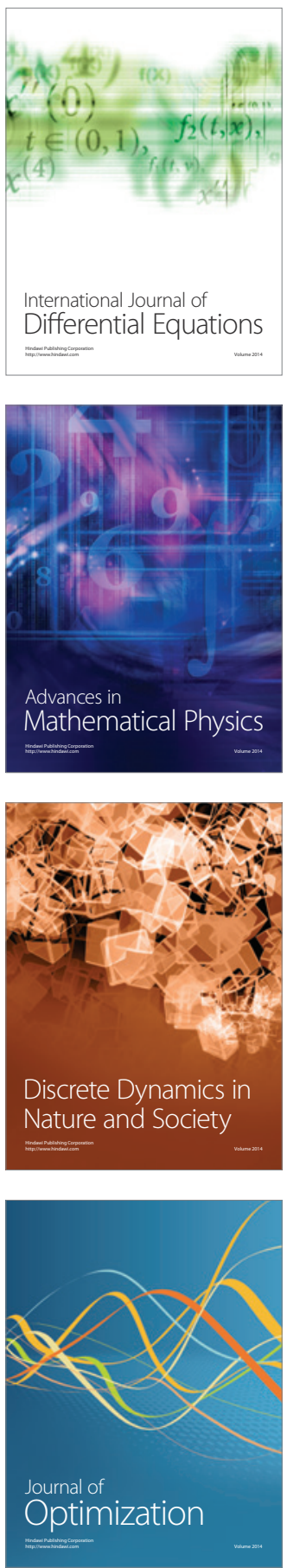